\title{
Morphology with a Null-Interface
}

\author{
Harald Trost and Johannes Matiasek \\ Austrian Research Instilute for Artificial Intelligence* \\ Schottengasse 3, A-1010 Vienna, Austria. \\ $\{$ harald, john\}oai.univie.ac.at
}

\begin{abstract}
We present an integrated architecture for word-level and sentence-level processing in a unification-based paradigm. 'The core of the system is a CLP implementation of a unification engine for feature structures supporting relational values. In this framework an IIPSG-style grammar is implemented. Word-level processing uses $\mathrm{X} 2 \mathrm{Mon} F$, a morphological component based on an extended version of two-level morphology. This component is tightly integrated with the grammar as a relation. The advantage of this approach is that morphology and syntax are kept logically antonomous while at the same time minimizing interface problems.
\end{abstract}

\section{Introduction}

Over the last few years there has been a growing interest in computational morphology and phonology. $\Lambda$ number of systems have been developed that deal with word-level processing. $\Lambda$ widely used approach is finite-state morphology, most notably two-level morphology (for an introduction, see Sproat 92). Morphological components are successfully used for a wide range of stand-alone applications like spelling correction and hyphenation. One obvious application is the use in NIP systems geared to the analysis/gencration of text. Surprisingly, they have not been widely applied in this domain up to now.

A major reason for this is the problem of interfacing morphology with syntax. Reflecting the current trend in syntax towards lexicalism, unification-based systems use highly structured feature structures as input. Translating the out put of morphological components into such a representation has proved to be diflicult. Reducing interface problems is therefore crucial to success.

- Financial support for the Austrian Rescarch Institute for Artificial Intelligence is provided by the Austrian Ministry of Science and Research. We would like to thank Wolfgang Ileinz for valuable comments and sugrestions
$A$ tight integration betwern word and sentence level processing also has linguistic advantages. The boundary between morphology and syntax is fuzzy. When processing written text the units morphology has to doal with are, in a technical sense, not words but character strings separated by delimiters. While these strings roughly correspond to the words of a sentence there are problematic cases. In Cierman, e.g., zu-infinitive or verbs with separable prefixes are written as a single unit in some instances and separately in others.

The problem has been recognized and some possible remedies lave been proposed. They all try to minimize or to eliminate the interface between word and sentence level processing. One step is the description of word formation in terms of a unification-based gramman to make the result of morphological processing directly available to syntax and vice versa, an approach already taken in X2MorF' ('Trost 90, 'Trost 91), an extension of two-level morphology.

The harder problem is the integration of morphophonology which is traditionally formalized in a way not easily translatable into the foature formalism. We will show how this can be achieved by merging the word-level grammar of X2MorF iuto an IIl'SCi-stylo grammar, and by adopting a rolational view of its two-level rules.

In this paper we assume basic familiarity with unifieation-based NLP' technicues and two-level morphology.

\section{Integrating Morphology into IPSG}

ILead-driven Phrase Structure Grammar (IIPSG, Pollard \& Sag 87, Pollard \& Sag in press) can be viewed as a mono-level but mulli-stratal theory of grammar, where different strata relate to diflerent aspects of linguistic information, but are represented miformly in feature logics. As such it is woll suited as a linguistic theory for our en- 
terprise.

IIPSG differentiates between three strataPHON, SYNSEM and DTRS. Though morphology is not considered in the standard approach, it suggests itself to be included as a fourth stratum by introducing a feature MORPI into the type sign. Morphotactics are easily described in terms of a feature based grammar. The problem is how to deal with morphophonology. Two proposals have been made to overcome this problem.

Krieger et al. 93 encode finite state antomata directly in the feature formalism. Since two-level rules can be compiled into such automata, morphophonology can be straightforwardly integrated into the grammar. While this is formally elegant it seems to be no good solution for practical considerations. First, it is not entirely clear from their paper how the problem of null characters can be handled. Second, encoding large automata will result in a very large and unwieldy type hierarchy. In general, introducing antomata into feature structures and encoding morphophonology directly at that level scems to be too low-level.

Bird \& Klein 93 argue against the use of two-level morphology because of linguistic considerations. The linguistic background of twolevel rules-main stream segmental phonologyhas widely been rejected as a valid linguistic model. Instead, they base their implementation on autosegmental phonology. (cf. Goldsmith 90).

This is certainly linguistically appealing. But there are reasons for sticking to a more conservative approach. Finite-state morphology as a formalism is not necessarily tied to segmental phonology. There are various approaches to cope with non-concatenative phenomena--one of them X2MorF (Trost 90). Also, for a number of languages complete sets of two-level rules do exist and can immediately be brought to bear. Finally, finite-state morphology has proven to be efficient while the method proposed by Bird \& Klein 93 seems to be computationally costly.

Like the other approaches ours is also based on IIPSG. However, we cmploy a different approach to integration. Our grammar is encoded using a unification engine based on constraint logic programming (CLP). Besides conventional attributevalue descriptions this system allows for the direct representation of more general relations, as they are required by IIPSG. This extension of the formalism is used for the integration of morphology. Thus X2MorF is treated as one special relation of the grammar. As a result, our approach is more modular than the others. While being fully integrated morphology can still be viewed as an autonomous component leading to a more flexible design.

We will now give an overview of X2MonF before describing the integrated system and its implementation in detail.

\section{Word Level Processing - $\mathrm{X} 2 \mathrm{MORF}$}

X2Mon P differs from standard two-level morphology in two important respects. Continuation classes are replaced by a feature-based word grammar. This allows for a more fine-grained description of morplıs. It is also a prerequisite for a tight integration with a unification-based grammar. X2MorF uses a morph lexicon where each morph has one or more feature structures assigned. The word grammar itself is simple. Morphs have a functor-argument structure along the lines of di Sciullo \& Williams 87. Aflixes are unary functors while stems are arguments without any further structure, resulting in a binary tree structure.

The other extension concerns the two-level rules, which are supplemented with a morphological filter consisting of a feature structure. This is important because in morphophonology only some rules are purely phonologically motivated. Oth. ers are triggered by a mixture of phonological and morphological facts. Such rules cannot be properly represented in the standard approach.

Take, e.g., mmlaut and scliwa epenthesis in German: The third person singular present tense suflix for German verbs is $-t$, e.g., sag-t $\rightarrow$ sagt. For stems ending in a dental, schwa is inserted before the ending, e.g., bad-t $\rightarrow$ badet. This rule does not hold across the whole vocabulary though. Stems of the strong paradigm do exhibit umlaut in 3rdPersigPres which blocks schwa epenthesis. The final dental of the stem must be omitted instead, e.g., rat-t $\rightarrow$ rïl.

The three rules ${ }^{1}$ shown in Fig. 1-together with the appropriate entries in the morph lexicon (cf. Iig. 7 below)-produce the required behavior. In particular, these rules relate surface rät to lexical $\$ n A t+b \$^{2}$. X2MorF can be seen as a re-

\footnotetext{
These rules as well as other data presented in the examples are simplified for the purpose of demonstration

2'The lexical character $A$ may have the surface realiza-
} 


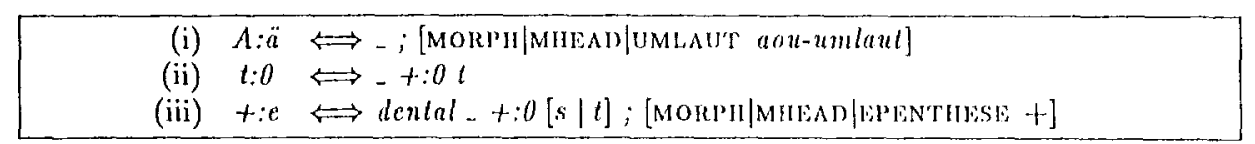

Figure 1: Three extended two-level Rules

lation between a surface string (the word form), a lexical string, and a feature structure (the interpretation of the word form). Relevant for sentence level processing is the morphosyntactic information and the stem, found as the values of paths MORPH|MIEAD and MORPH|STEM Tespectively (cf. Fig. 9 below). This is supplemented by lexrme specific information in the value of SYNSEM (for at detailed description see Trost 93).

\section{Implementing HPSG in a CLP Framework}

IIPSG employs strongly typed feature structures together with principles constraining them further. Well-typedness requirements restrict the space of valid feature structures (cf. Carpenter 92): Every feature structure must be associated with a type, and every type restricts its associated feature structure in that only certain features are allowed and the values of these features must be of a certain type. Appropriateness and value restrictions are in herited along the type hicrarchy.

The second source of constraints, in order to admit only linguistically valid feature structures, are the principles of grammar. Pollard \& Sag 87 allow general implicative and negative constraints in the form of conditional feature structures. In Pollard \& Sag in press principles are given only in verbal form. Recent work on formalizing the basis of IIPSG models them as constraints attached to types (e.g., Carpenter et al. 91). However, these distinctions affect only how the applicability of a principle is specified. More important for our present purpose is the form which the constraints expressed by a principle may take. Besides constraints enforcing simple structure sharing (e.g., the IIead Feature Principle given in Fig.2) there are also complex relational dependencies (e.g., in the Subcategorization Principle $\left.{ }^{3}\right)$. Constraints

tions $a$ and $\ddot{a}$. The rule has an empty phonological context but a morphological filter. This is an example for the treatment of nom-concatenative phenomena in X2Mor $\mathrm{F}^{\circ}$.

3 "In a headed phrase (i.e., a phrasal sign whose nTns value is of sort head-struc), the suncer value of the head like these go beyond the expressivity of pure feature formalisms alone and need to be defined in a recursive manner.

In order to integrate such complex constraints in the feature unification framework we interpret unification of typed feature structures under the restrictions of principled constraints as constraint solving in the (IJl' paradigm (Jaflar \& I Jassez 87).

In CLP the notion of unification is replaced by the more general notion of constraint solving. Constraint solvers may be embedded into a logic programming language either by writing a metainterpreter or by making use of a system which allows for the implementation of mification extensions.

The sncond approach is taken by DMCAI (J) $1^{4}$ (Jolzbaur 92), a l'rolog system whose unification mechanism is extended in such a way that the user may introduce interpreted terms and specify their meaning with regard to unification through Prolog predicates. The basic mechanism to achieve this behavior is the use of attributed variables, which may be qualified by arbitrary user-defined atributes. Attributed variables behave liko ordinary Prolog variables with two notable excoptions; when an attributed variable is to be unified with a non-variable term or another attributed variable the mification extensions come into play. For ejther case the user has to supply a predicate which explicitly specifies how the attributes interact and how they should be interpreted with respect to the semantics of the application domain. Unification succeds only if these constraint solving clauses managing the combination-or verification-of the involved attributes are successful.

The implementation of typed feature structures in our system makes use of the CIP facilities provided by this enhanced Prolog system. Feature structures are implemented by the attribute

daughter is the concatenation of the phrase's SUBCAT list with the list (in order of increasing obliqueness) of SYNSLM values of the complement clanghters." (Pollard \& Sag in press)

'I)MCAI CLL' is an enthanced version of SICSStus Prolog, available by anonymous flp from ftp, ai.univie.ac at 
Is (Type, Dag, Goals), where Dag is a list of featurevalue pairs (which is empty in case of atomic types) or a marker indicating uninstantiatedness of the substructure (feature structures are instantiated lazily). Goals is a list of delayed constraints (see below). Well-typed unification of two feature structures is implemented via the constraint solving clauses mentioned above, taking into account type hierarchy and feature appropriateness (for a detailed description ef. Matiasek \& IIeinz 93).

Constraints imposed onto feature structures by the principles of grammar are stated in a conditional form where the antecedent is restricted to contain only typing requirements. ${ }^{5}$ In order to account for these conditional constraints we adopt a licensing view: Every node of a feature structure has to be licensed by all principles of grammar.

A node is licensed by a principle if either (i) the feature structure $F$ rooted in that node satisfies the applicability conditions of the principle and the constraints expressed by the principle successfully unify with $F$, or (ii) the feature structure $F$ rooted in that node is incompatible with the applicability conditions of the principle. The interesting case arises when a feature structure does not satisfy the applicability conditions of the principle but is compatible with them. Thus applicability of the principle can be decided only later, after further instantiation or unification steps have restricted the (sub)structure rooted at that node. In precisely this case the application (or the abandoning) of the constraint has to be delayed. The delay mechanism utilizes the Goals slot in the $\mathrm{fs} / 3^{6}$ attribute, which is derlicated to hold the delayed constraints. As an example take the well known Ilead Feature Principle of IPSG (Fig. 2$)^{7}$. The conditional operator $==\Rightarrow$ is translated at read time via term_expansion $/ 2$ and implements the delay mechanism by compiling precondition checks into the principle. These antecedent chocks trigger either the application of the principle, its abandonment, or its delay (by annotating the variables which are not sufficiently constrained to decide on the antecedent with the delayed goals).

'Two advantages of this approach to implement

\footnotetext{
${ }^{5}$ This is only a syntactic variant of attaching constraint solely to types (Carpenter et al, 91) and does not permit general conditional structures as used in Pollard \& Sag 87.

${ }^{6}$ pred $/ \mathrm{n}$ is the usual notation for a n-ary Prolog predicate.

TThe opcrators $:: *,::,:,= \pm$ are defined for typing of a node, path restriction, patl concatenation and value restriction (type or coreference) respectively.
}

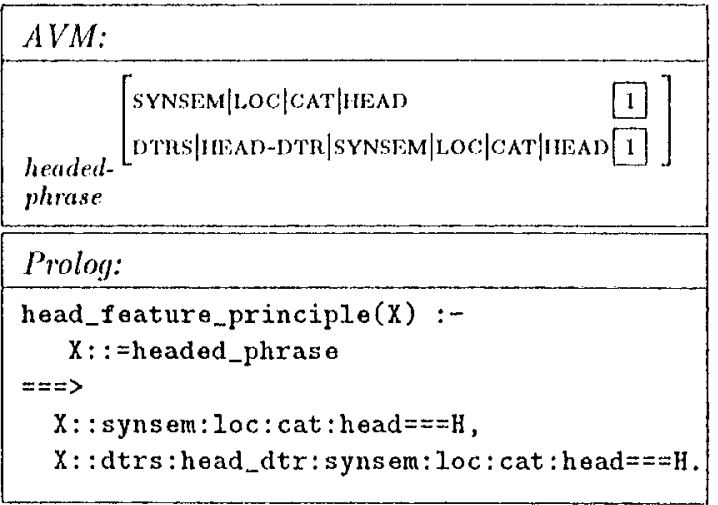

Figure 2: Head Feature Principle

principled constraints are especially important for our present purpose: First, stating redundant typing requirements for embedded structures (i.e. type restrictions that would follow antomatically from well-typing) forces delay of the conditional constraint until these substructures are instantiated. This device can, e.g., be used to block infinite recursion in recursively defined constraints. Second, the right hand part of the conditional is not restricted to leature logical expressions, but instead can contain arbitrary Prolog goals. In this way constraints involving relational dependencics (such as the Subcategorization Principle and the morphological relation between a lexical and a surface string) can be expressed within the feature formalism and there is no need for external devices controlling this interaction. Furthermore, the conditional constraint syntax is not restricted to unary licensing principles but can also be used to express relations, such as is_append/3-needed for implementing the Subcat Principle-which appends two foature structure lists (Fig. 3). Note

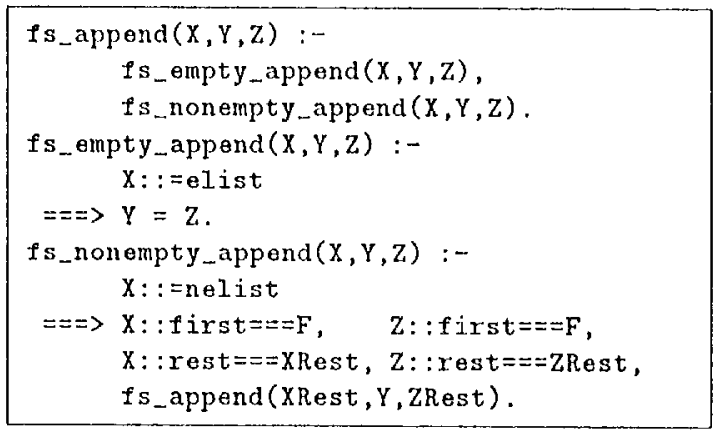

Figure 3: $\Lambda$ ppend for feature struclure lists

that disjunctive relations such as append can now be written as the conjunction of two specialized cases applying conditionally. Furthermore, in- 


\begin{tabular}{|c|c|}
\hline & $t: 0 \leftrightarrow-+: 0 t$ \\
\hline Inpul & $-\Leftrightarrow t: 0 \Leftrightarrow\left[t^{\prime}: 0, t: t\right]$. \\
\hline Compiled & $\begin{array}{l}\text { morphrule }([116,43,116 \mid \mathrm{LS}],[\mathrm{Sc}, 18,116 \mid \mathrm{SS} 0], \text { SS , LCon, SCon, } F):- \\
\text { !, Sc=48, } \\
\text { morphology }([43,116 \mid \mathrm{LS}],[48,116 \mid \mathrm{SSO}], \mathrm{SS},[116 \mid \mathrm{LCon}],[\mathrm{H} \mid \mathrm{SCon}], \mathrm{F}) \text {. }\end{array}$ \\
\hline
\end{tabular}

Figure 4: Sample Two-Level Rule

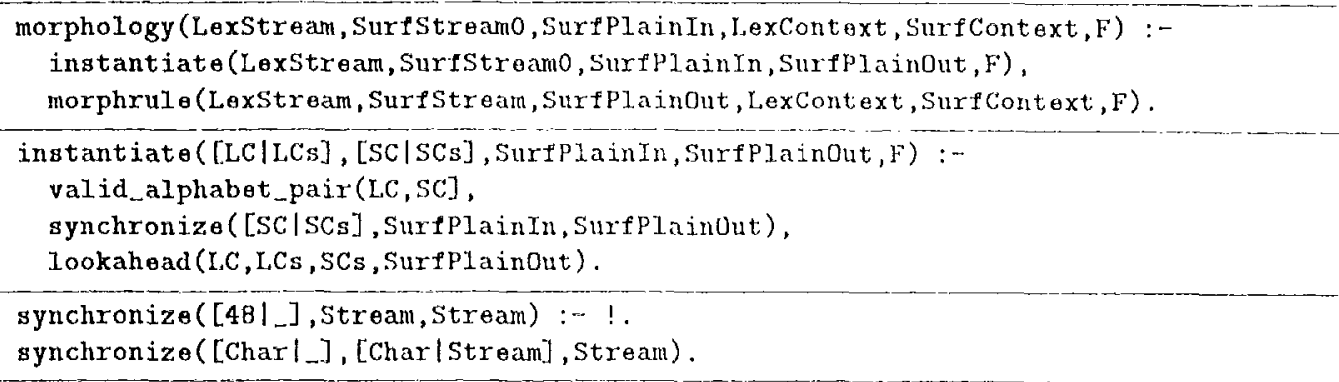

ligure 5: The morphology relation

finite loops due to uninstantiated variables can never occur, a crucial requirement when integrating relational dependencies into a lazy instantiating feature formalism.

\section{$5 \quad$ Embedding X2MorF into the Feature System}

Originally $\mathrm{X} 2 \mathrm{MonF}$ was realized as a separate morphological component interfaced to the sentence analyzer/generator only via sequential data transfer. In the case of analysis, the feature structure representing the word form was transmitted to the parser. For generation, X2Morl' expected a feature structure as input reproducing one or more word forms. This purely sequential architec. ture was not satisfactory because of the problems mentioned in the introduction.

In order to achieve tight integration, we adopt a relational view of $X 2 \mathrm{M}$ on $\mathrm{F}$ and encode the relation between surface string and lexical string directly without using finite state aulomata (for arguments supporting this approach cf. Abramson 92). Ilowever, our approach extends $\Lambda$ bramson 92 in that it (i) explicitly accounts for the insertion of null characters and (ii) introduces the filter concept of $X 2 \mathrm{Mon} F$ into the relational approach.

The general format of a two-level rule specilication in our system is

LCon $\Leftrightarrow$ Transition $\Leftrightarrow$ RCon [:- Filter]

in the case of equivalence rules, optional rules are written using only single arrows $(\Rightarrow$ and $\Leftrightarrow=$ ).
These rules are compiled into Prolog clauses 8 relating the lexical and surface clatacter streans appropriately (see lige.t for an example of the $t$ elision rule for (ierman).

To obtain a correct relationship between surface and lexical string every transition has to be licensed by a morphological rule. 'Transitions not mentioned by rules are handled by a default rule. Instantiation of contexts may not be done by the rules themselves, since this would make it impossible to obtain negation via the cut-operator. Instead, it is handled separalely in a backtrackable fashion.

The cental relation is the morphology predicate, (see ligk, 5) moliating between lexical string, surfare string (with inserted null elements), the pure (droullified) surface string and the feature structure of the morphological sign. Instautiation of pairs is done depending on the possible lexiral continuations (the lexicon being represented by a trie-structure). The amomnt of lookahead is determined by the current pair which is to be licensed by morphrule. Synchronization of surface and lexical string by insertion of mull characters is also haudled at the instantiation level.

The integration of the two-level relation into

\footnotetext{
"Nole: that leit contexts are ancoded reversed to ac count for the left to right traversal of the pair of character streams. lueft contexts an be remembered and checked most efficiently this way.

This interaction and the lexicon looknp of the feature structure corresponding to the current morph, which takes place when encomutering a morple boundary is not shown for the siake of simplicity.
} 
the general framework of the feature based sentence-level and word-level grammars is now performed by adding this relation as a principled constraint at the appropriate level.

In a definite clause style $A V M$ notation this could be written as follows (given that morphology/3 is a wrapper around the morphology relation given above, starting with empty left context and hiding the nullified surface stream):

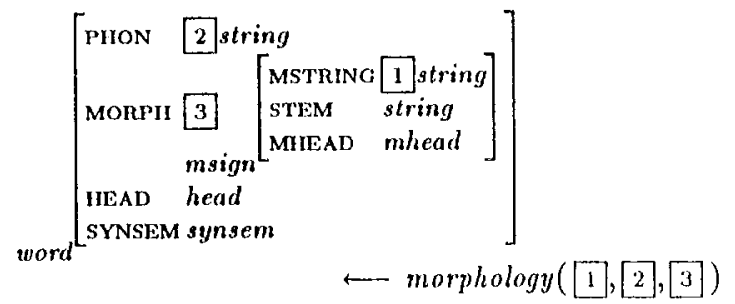

The actual implementation as a principled constraint in our formalism additionally takes care of delaying the actual enforcement of this relation in case the strings are not sufficiently instantiated.

A second provision has to be made in the word level grammar to assure proper concatenation of the lexical strings of the morphological signs being combined. Given the subtyping of msign into marg and mfunctor, which in turn has the subtypes lefifunctor and rightfunctor, the principled constraints ensuring concatenation of a left functor with its argument are shown in Fig. 6. Concatenation is delayed until the ar-

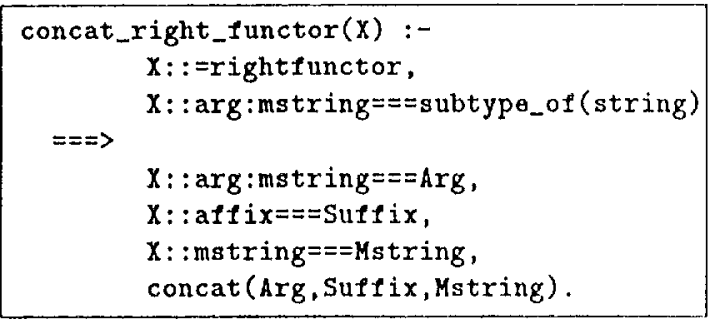

Figure 6: Concatenation of lexical strings

gument's MSTrING is instantiated. Thus, infinite loops when concatenating are avoided.

As an example we demonstrate how these constraints interact in forming the third person singular present tense form of the German verb riten (to guess). The lexical string is composed of the stem $r A t$ and the suffix $+t$. The lexical entries of these two morphs are given in Fig. 7.

The two-level rules applicable for this example are the t-elision rule (Fig.4) and two rules with filters licensing a-umlaut and epenthesis, given in

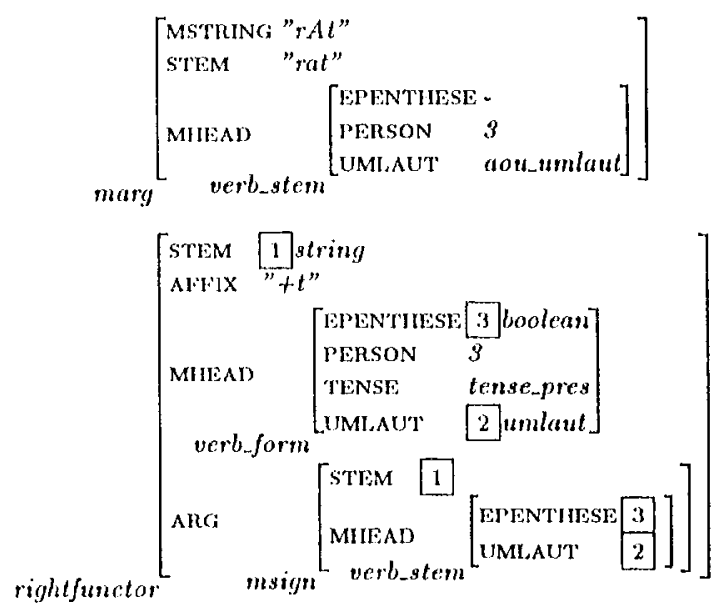

Figure 7: Lexical entries

the input notation for our system (Fig.8).

Interaction between syntactic and morphological processes takes place at the word level. The application of the two-level rules relating the surface string (i.e the pron-value of the word) and the lexical-string (i.e. MORPIIMSTRING) is also triggered here. This interaction is completely neutral with respect to the direction of processing due to its relational nature. Parsing is performed by simply instantiating the PHON value. Generation can be achieved when MORPII|MSTRING is present, which in turn is obtained by concatenating the lexical strings of the msigns instantiated by the morpli grammar.

As a result of this constraint interaction the structure shown in Fig. 9 is obtained. Features relevant at the syntactic level (such as PERSON and TFNSE) are percolated from MORPI/MHFAD to SYNSEM|IOC|CAT|IIEAD via structure sharing constraints attached to the type word (this interaction is not shown in Fig. 9). Information on subcategorization and semantic content for the word is obtained from the lexeme lexicon using MORPIISTEM as a key. These constraints complete the interaction between syntactic and morphological processing at the word-level.

\section{Conclusion}

We have presented a framework for the tight integration of word level and sentence level processing in a unification-based paradigm. The system is built upon a unification engime implemented in a CLP language supporting types and definite relations as part of feature descriptions. Using this ex- 


\begin{tabular}{|c|c|}
\hline A-umlaut & $-\Leftrightarrow A: " a \Leftrightarrow-:-f i l t e r(X,[X::$ mhead: umlaut $===$ aou_unlaut $])$ \\
\hline lipenthesis & dental $\Leftrightarrow{ }^{+}: \theta \Leftrightarrow$ s_or.t : - filter $(X,[X:$ mhead: epenthese==='+']) \\
\hline
\end{tabular}

Figure 8: Filter Rules

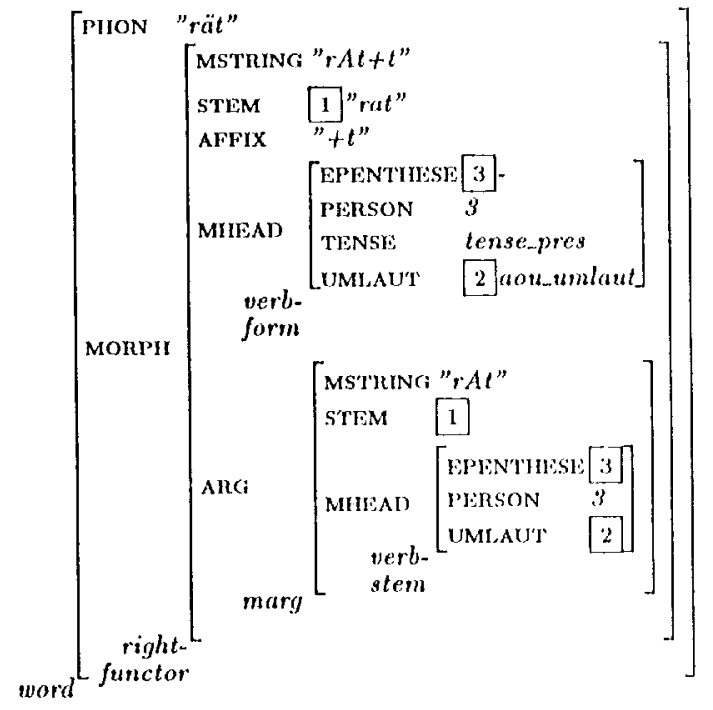

Figure 9: Result of constraint interaction

tended feature formalism, which is independently motivated by requirements of standard $I P S G$, a reimplementation of $\mathrm{X} 2 \mathrm{Mon} \mathrm{F}$ was integrated into the grammar as a specialized relation.

This architecture has computational as well as linguistic advantages. Integrating morphology and morphophonology directly into the grammar is in the spirit of HPSC, which views grammar as a relation between the phonological (or graphemic) form of an utterance and its syntactic/semantic representation. This way the treatment of phenomena transcending the boundary betwen morphology and syntax is also made possible.

On the implementation side, the pratical problems of interfacing two inherently diflerent modules are climinated. For applications this means that using a morphological component is made easy. Nevertheless, this tight integration still leaves morphology and syntax/semantics as autonomous components, enabling direct use of existing data sets describing morphophonology in terms of the two-level paradigm.

\section{References}

Abramson H.: A Logic Programming View of lielational Morphology, in Proceedings of the $15 \mathrm{th}$
COIING, August 23-28, 1992, Vol.III, pp.850-854, 1992 .

Bird S., Klein P.: Fnriching IJPSG Phonology, University of lidinburgh, UK, Research Paper PUCCS/RP'-56, 1993.

Carpenter B., Pollard C., Franz A.: The Specification and Implementation of Constraint-Based Unification Cirammars, Proccedings of $2^{\text {nd }}$ IWP'T, Cancun, Mexico, 143-153, 1991.

Carpenter B.: The Logic of Typed Fealure Structures, Cambridge University l'ress, Cambridge Tracts in 'Theoretical Computer Science 32, 199\%.

Goldsmith J.A.: Aulosegmental and Metrical Phonology, Basil Blackwell, Oxford, 1990.

Ilolzbaur O.: Metastructures vs, Attributed Variables in the Context of lixtensible Unification, in Bruynooghe M. and Wirsing M.(eds.), Programming Language Implementation and Logic Programming, Springer, LNCS 631, pp.260-268, 1992.

Jaflar J., Latsse\% J.L.: Constraint, Logic Progranming, in Proceelings l4th ACM POPI, Conf., Munich, 1987.

Krieger II.-U, Pirker II., Nerbonne J.: Feature-based Allomorphy, Proceedings of the 31 Ist Annual Meeting of the $\Lambda$ (CL, Columbus, Olio, pp.140-147, 1993.

Matiasek J., Heinz W.: A CLP Based Approach to IIPSC, Osterreichisches Porschungsinstitut fïr $\Lambda \mathrm{r}$ tificial futelligence, Wien, TR-93-26, 1993.

Pollard (..)., Sag I.A.: Informalion-Based Synlax and Semamlics, University of Chicago Press, Chicago, 1987.

Pollard, (:.), Sag I.A.: Head-Driven Phrase Siructure Grammar, University of Chicago Press and CSLl Publications, in press.

di Sciullo A.-M., Williams li.: On the Definition of Word, Ml'l P'ress, Cimblerdge, MA, 1987.

Sproat R.: Morphology and Compulation, MIT Press, Cambridge, MA, ACI,-MIT Series in NL]', 1992.

'Trost II.: 'The Application of Two-Level Morphology to Non-Concatenative Cerman Morphology, in Karlgren Il.(ed.), Procedings of the 13th COLING, Ilelsinki, Finland, pp.371-376, 1990.

'Thost HI: X2MORF: A Morphological Component Based on Augruented Two-Level Morphology, in Procedings of the 12 h IJCAI, Morgan Katurnann, San Mateo, CA, pp.1024-1030, 1991.

'Trost I1.: (ophing, with Derivation in a Morphological Component, in 6th Conference of the European Chater of the $A(\mathrm{t}$, Utredt, pp.368-376, 1993. 